\title{
Measurements of the changes in angular diameter of Mira variables with pulsation phase
}

\author{
J. S. Young, J. E. Baldwin, R. C. Boysen, C. A. Haniff, D. Pearson, \\ J. Rogers, D. St-Jacques, P. J. Warner and D. M. A. Wilson \\ Mullard Radio Astronomy Observatory, Cavendish Laboratory, \\ Madingley Road, Cambridge CB3 OHE, UK
}

\begin{abstract}
.
We report the direct detection of cyclic diameter variations in the Mira variable $\chi$ Cygni. Interferometric observations made between 1997 July and 1998 September, using the Cambridge Optical Aperture Synthesis Telescope (COAST) indicate periodic changes in the apparent angular diameter with amplitude 45 per-cent of the smallest value.

The measurements were made in a $50 \mathrm{~nm}$ bandpass centred on $905 \mathrm{~nm}$, which is only moderately contaminated by molecular absorption features. To assess the effects of atmospheric stratification on the apparent diameter measured in this band, we have also measured near-infrared diameters for a sample of five Miras, in both the J-band $(1.3 \mu \mathrm{m})$ and Wing's (1971) $1.04 \mu \mathrm{m}$ band, which is expected to isolate essentially pure continuum emission. We present J-band visibility curves which indicate that the intensity profiles of the stars in the sample differ greatly from each other.
\end{abstract}

\section{Introduction}

Diameter changes of Mira-variable stars have been measured previously by van Belle et al. (1996), who found variations with phase within a sample of one or two-epoch diameter measurements of 18 stars, and by Tuthill, Haniff \& Baldwin (1995), who made seven observations of $o$ Ceti spread over a three year period.

Cyclic variations in the apparent diameter of an individual Mira were first detected by Burns et al. (1998, henceforth B98), who monitored R Leonis with COAST (Baldwin et al. 1998) and the William Herschel Telescope for almost two pulsation cycles.

In this paper we present COAST diameter measurements of $\chi$ Cygni, which exhibit phase-coherent changes similar to the variations previously seen in $\mathrm{R}$ Leonis. 


\section{Phase-coherent diameter changes}

\subsection{Observations}

$\chi$ Cygni was monitored with COAST at two wavelengths: $905 \mathrm{~nm}$ and $1290 \mathrm{~nm}$. Observations were made on 22 nights (between the dates of 1997 July 20 and 1998 September 19), covering a full pulsation cycle. The observation and data reduction procedures are outlined by Burns et al. (1997).

\subsection{Results and discussion}

In order to characterise the stellar size, we have fitted both uniform disk and Gaussian functions to the visibility amplitude data. At a wavelength of $905 \mathrm{~nm}$, a Gaussian was a better fit to the long baseline data $(V<30 \%)$, even near maximum light, and so only the results of the Gaussian fits are presented here. However, the phase-coherent modulation seen in the Gaussian diameters at $905 \mathrm{~nm}$ is also seen in the $905 \mathrm{~nm}$ uniform disk diameters, which suggests that most of the variation is due to a real change in the scale of the emitting region.

The apparent angular diameter of $\chi$ Cygni at $905 \mathrm{~nm}$ (Fig. 1) clearly varies in a phase coherent manner: the star appears smallest at visual phase 1.0 and largest near phase 0.5, the same as B98 found for R Leonis (see Fig. 2). The 1992 measurement of Haniff, Scholz \& Tuthill (1995) is consistent with the diameter modulation seen during 1997 and 1998 and therefore suggests that the variation in $\chi$ Cygni may be coherent over five cycles. The peak-to-peak amplitude of the variation is approximately $45 \%$ of the smallest diameter. The amplitude of any variation at $1.3 \mu \mathrm{m}$ is much smaller. B98 found that the diameter of $\mathrm{R}$ Leonis varied by $\sim 35 \%$ at both $830 \mathrm{~nm}$ and $940 \mathrm{~nm}$.

The flux transmitted by the 830,905 and $940 \mathrm{~nm}$ filters is dominated by continuum emission, with only a small amount of atomic and molecular blanketing. There is however weak contamination by $\mathrm{TiO}$ absorption bands, whose strength varies with pulsation phase (Spinrad \& Wing 1969). Hence the detected photons originate from several different layers within the stratified photosphere of the star, whose relative contributions vary with phase. It is clear that the diameter we measure in these wavebands does not simply tell us the position of the continuum-forming layers.

Hofmann, Scholz \& Wood (1998) have calculated the apparent diameters of six model Mira variables covering a wide range of effective temperatures, at various wavelengths and pulsation phases. The physical size of their model stars is largest at phase $\sim 0.2$, therefore the apparent diameter in the continuum is larger at maximum than at minimum light for all six of the model series. At near-continuum wavelengths increased contamination by molecular bands tends to enlarge the star near minimum. Despite this effect, five of the six model series still appear larger at maximum, the exception being the $\mathrm{P}$ series (low- $T_{\text {eff }}$, fundamental-mode pulsation), whose apparent diameter at $820 \mathrm{~nm}$ and $920 \mathrm{~nm}$ is $15-30 \%$ larger at minimum than at maximum. The change in our Gaussian fit between minimum and maximum should be slightly smaller than this, if the Hofmann et al. predictions for phase-dependent limb-darkening are correct. These differences are much less than the $45 \%$ we have observed for $\chi$ Cygni at $905 \mathrm{~nm}$, which may suggest that the treatment of molecular opacity in the models is inadequate. 

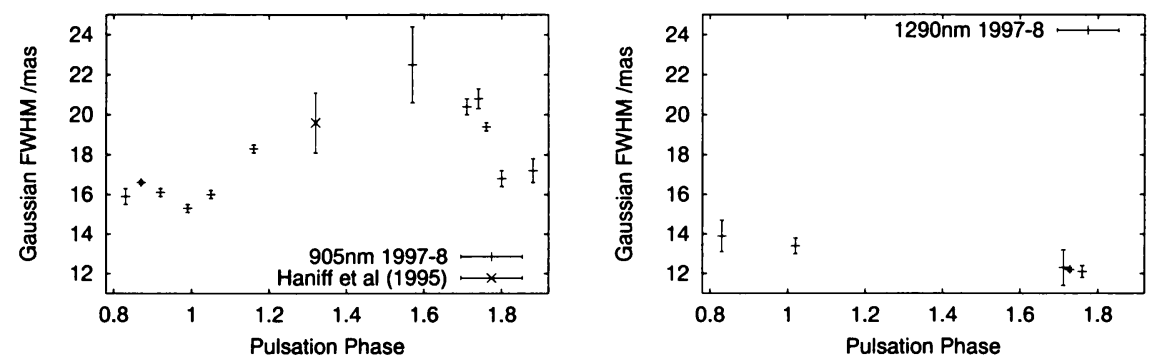

Figure 1. Apparent angular diameters (Gaussian full widths at halfmaximum intensity) and $1 \sigma$ errors for $\chi$ Cygni at $905 \mathrm{~nm}$ (left) and $1290 \mathrm{~nm}$ (right), plotted against the phase of the visual light curve (zero is maximum). The diagonal cross at phase 1.32 is the 1992 measurement at $902 \mathrm{~nm}$ of Haniff, Scholz \& Tuthill (1995). The diameter increases slowly from phase 1.0 to phase $\sim 1.5$, then decreases rapidly between phases 1.6 and 1.8. A similar slow diameter increase during the first half of the pulsation cycle is seen in the B98 measurements of $\mathrm{R}$ Leonis (Fig. 2).
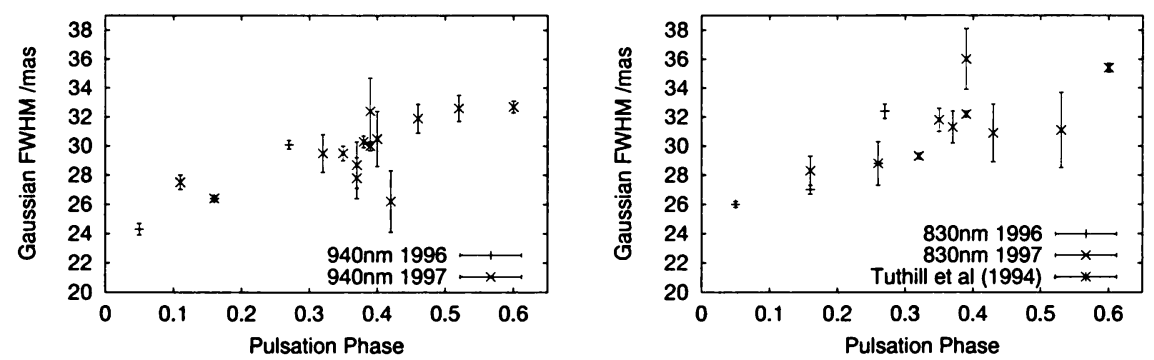

Figure 2. Apparent angular diameters (Gaussian full widths at halfmaximum intensity) and $1 \sigma$ errors for $\mathrm{R}$ Leonis at $940 \mathrm{~nm}$ (left) and $830 \mathrm{~nm}$ (right), plotted against the phase of the visual light curve (zero is maximum). Horizontal marks are 1996 data, and diagonal crosses are 1997 data. The 1996 and 1997 points are the result of Gaussian fits to the visibility amplitude measurements of B98 (who plot the results of uniform disk fits to the same data). The asterisk at phase 0.25 is the 1992 measurement of Tuthill et al. (1994). Note the different horizontal scale from Fig. 1. 

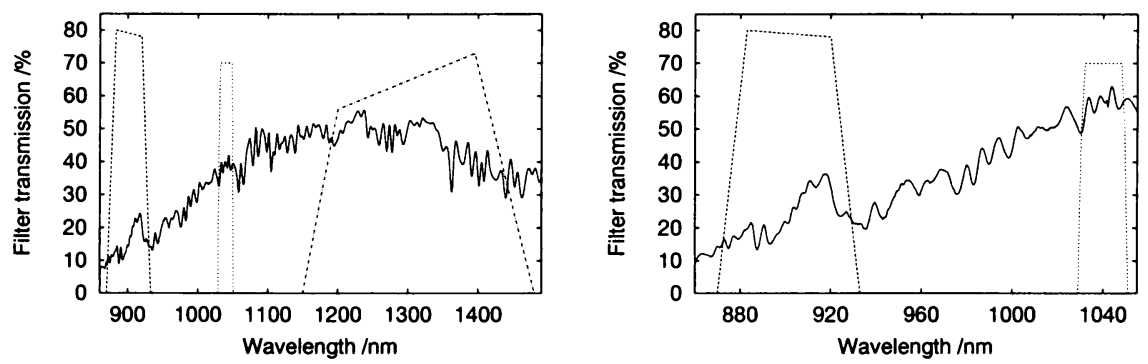

Figure 3. Wavebands used for observations, superimposed on the spectrum of an M8 giant star, from Pickles (1998). From left to right, the centre wavelengths and half-power bandwidths of the filters are $905(50) \mathrm{nm}, 1040(19) \mathrm{nm}$ and $1290(260) \mathrm{nm}$. The right plot shows the shorter-wavelength region in more detail.

\section{Variation of apparent diameter with waveband}

In order to determine the effect of atmospheric stratification on apparent diameters measured in different wavebands, we have begun a programme of diameter measurements in two bands where we expect most of the radiation to originate from a single layer: Wing's $1.04 \mu \mathrm{m}$ continuum band (Wing 1971), and the $\mathrm{J}$-band at $1.3 \mu \mathrm{m}$. Contemporaneous measurements were made in the $905 \mathrm{~nm}$ band. The filter bandpasses are shown schematically in Fig. 3. We have observed a sample of five Mira variables in these bands.

For those targets where we have data on a range of baselines, we find:

- Visibility amplitudes at $905 \mathrm{~nm}$ are all well fit by a Gaussian.

- At $1.3 \mu \mathrm{m}$ a Gaussian model is generally a poor fit to the long-baseline data, but there are some striking differences between different stars in the sample, as illustrated by the visibility curves shown in Fig. 4.

In order to compare measurements at different wavelengths, we fitted a Gaussian model even when this model was a poor fit at long baselines. In such cases the long baseline visibilities were removed. We found that the apparent Gaussian FWHM varies substantially with wavelength. The scale of the variations is clearer if we normalise the $905 \mathrm{~nm}$ and $1040 \mathrm{~nm}$ diameters to the $1.3 \mu \mathrm{m}$ diameter (which varies least with phase); these ratios are given in Table 1.

The apparent diameter at $905 \mathrm{~nm}$ is always larger than that at $1.04 \mu \mathrm{m}$ or $1.3 \mu \mathrm{m}$, probably due to absorption by $\mathrm{TiO}$ in the outer atmosphere of the star. The ratio of the diameter at $905 \mathrm{~nm}$ to that at $1.3 \mu \mathrm{m}$ appears to be phasedependent.

The $1.04 \mu \mathrm{m}$ continuum band diameters are also all larger than the J-band diameters. This may be due to a combination of the following:

- Molecular absorption in the outer atmosphere (which leads to a larger diameter being measured). 

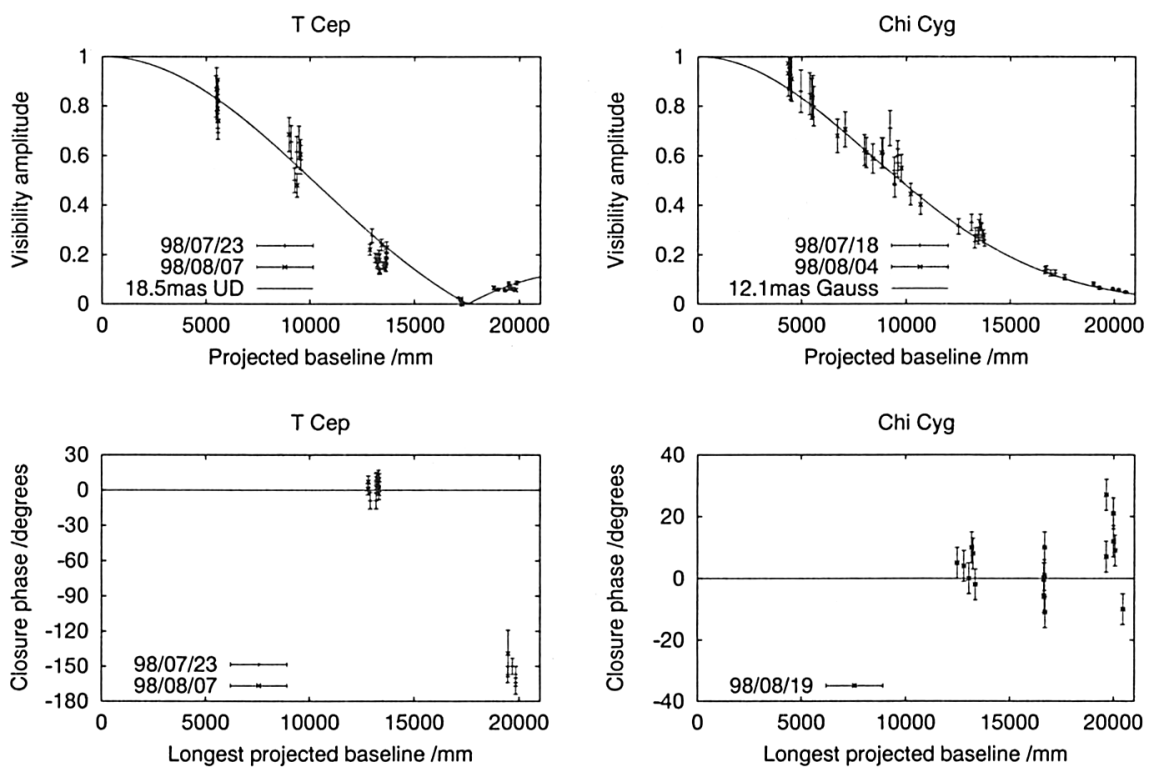

Figure 4. J-band visibility curves (top) and corresponding closure phases (bottom) for T Cephei (left) and $\chi$ Cygni (right). The visibility amplitude for $\mathrm{T}$ Cephei goes through a zero, and at the same baseline the closure phase changes from 0 to $\sim 180$ degrees, as expected for a centro-symmetric disk. There is evidence for some limb-darkening. In contrast, there is no null in the visibility function for $\chi \mathrm{Cygni}$, which is very well fit by a Gaussian model for the intensity profile.

Table 1. Ratio of apparent angular diameter $D_{\lambda}$ (Gaussian FWHM) at $905 \mathrm{~nm}$ and $1040 \mathrm{~nm}$ to that at $1290 \mathrm{~nm}$. The apparent diameter at $1290 \mathrm{~nm}$ is given in the 6 th column.

\begin{tabular}{lllllc}
\hline Star & Date(s) & Phase & $D_{905} / D_{1290}$ & $D_{1040} / D_{1290}$ & $D_{1290}$ \\
\hline$\chi$ Cyg & $97 / 07 / 19,20$ & 0.83 & $1.14 \pm 0.07$ & & $13.9 \pm 0.8$ \\
& $97 / 09 / 22,97 / 10 / 05$ & 1.00 & $1.14 \pm 0.04$ & & $13.4 \pm 0.4$ \\
& $98 / 07 / 18,24$ & 1.73 & $1.72 \pm 0.04$ & $1.57 \pm 0.04$ & $12.1 \pm 0.1$ \\
& $98 / 08 / 04$ & 1.76 & $1.60 \pm 0.04$ & & $12.1 \pm 0.3$ \\
& $98 / 08 / 04,18$ & 1.78 & $1.39 \pm 0.05$ & $1.14 \pm 0.06$ & $12.1 \pm 0.3$ \\
R Cas & $97 / 10 / 18,22$ & 0.52 & $1.32 \pm 0.14$ & & $16.1 \pm 0.6$ \\
& $98 / 08 / 02,05$ & 1.19 & $1.15 \pm 0.02$ & & $16.6 \pm 0.2$ \\
T Cep & $98 / 08 / 02,07$ & 0.23 & $1.12 \pm 0.03$ & & $13.7 \pm 0.2$ \\
& & & & \\
o Cet & $98 / 08 / 10,18,19$ & 0.67 & $1.34 \pm 0.03$ & $1.16 \pm 0.03$ & $19.4 \pm 0.3$ \\
& & & & & \\
T Cas & $98 / 08 / 18,19$ & 0.60 & $1.21 \pm 0.04$ & $1.06 \pm 0.05$ & $7.8 \pm 0.2$ \\
\hline
\end{tabular}


- Wavelength-dependent continuous opacity (an increase in opacity leads to a larger diameter being measured).

- Wavelength-dependent limb-darkening (increased limb-darkening makes the star appear smaller).

We will consider molecular absorption first. Our $1.04 \mu \mathrm{m}$ bandpass may be contaminated by VO, but the VO band only covers at most $10 \%$ of the filter bandpass, so any increase in the observed apparent diameter due to VO will be

small. A similar effect may enlarge the J-band diameter: the spectra of cool stars have a wide absorption feature at $1.4 \mu \mathrm{m}$ due to $\mathrm{H}_{2} \mathrm{O}$. However, the increase in apparent diameter will be small because most of the photons in the wavelength range of the feature will be absorbed by telluric water and thus not detected.

It therefore seems unlikely that the observed apparent diameter ratios can be explained by spectral features. The dominant source of near-infrared continuous opacity in cool stars is $\mathrm{H}^{-}$, and the total $\mathrm{H}^{-}$opacity is greater at $1.04 \mu \mathrm{m}$ than at $1.3 \mu \mathrm{m}$ (Wing \& Rinsland 1981). This, together with the effect of small changes in limb-darkening, may explain the observed diameter ratios.

\section{Conclusions}

We have measured phase-coherent variations in the apparent diameter of $\chi$ Cygni. The amplitude at a wavelength of $905 \mathrm{~nm}$ is $45 \%$ of the smallest diameter.

We have observed a sample of five Miras in two other, less-blanketed nearinfrared bands. Brightness profiles inferred from J-band data show striking differences between the sample members, and the J-band diameters are systematically smaller than those measured in the $1.04 \mu \mathrm{m}$ continuum window.

Our results provide important new constraints for theoretical models of Mira variables.

\section{References}

Baldwin J.E., et al., 1998, Proc. SPIE 3350, 736

Burns D., et al., 1997, MNRAS 290, L11

Burns D., et al., 1998, MNRAS 297, 462 (B98)

Haniff C.A., Scholz M., Tuthill P.G., 1995, MNRAS 276, 640

Hofmann K.-H., Scholz M., Wood P.R., 1998, A\&A 339, 846

Pickles A.J., 1998, PASP 110, 863

Spinrad H., Wing R.F., 1969, ARA\&A 7, 249

Tuthill P.G., Haniff C.A., Baldwin J.E., Feast M.W., 1994, MNRAS 266, 745

Tuthill P.G., Haniff C.A., Baldwin J.E., 1995, MNRAS 277, 1541

van Belle G.T., Dyck H.M., Benson J.A., Lacasse M.G., 1996, AJ 112, 2147

Wing R.F., Rinsland C.P., 1981, Rev. Mexicana Astron. Astrof. 6, 145

Wing R.F., 1971, in Proc. Conf. on Late-Type Stars (Kitt Peak Natl. Obser. Contr. No. 554), G.W. Lockwood and H.M. Dyck (eds.), p. 145 